\title{
Cancer-educated mesenchymal stem cells promote the survival of cancer cells at primary and distant metastatic sites via the expansion of bone marrow-derived-PMN-MDSCs
}

\author{
Buqing Sai 1,2,3,4, Yafei Dai 1,2,3, , Songqing Fan ${ }^{5}$, Fan Wang ${ }^{1,2,3,4}$, Lujuan Wang ${ }^{1,2,3,4}$, Zheng Li 1,2, Jingqun Tang ${ }^{6}$, Li Wang ${ }^{6}$, \\ Xina Zhang ${ }^{1,2,3,4}$, Leliang Zheng ${ }^{1,2,3,4}$, Fei Chen ${ }^{7}$, Guiyuan $\mathrm{Li}^{1,2,3,4}$ and Juanjuan Xiang $\mathbb{B D}^{1,2,3,4}$
}

\begin{abstract}
Bone marrow mesenchymal stem cells (BMSCs) are multipotent stromal cells that can differentiate into a variety of cell types. BMSCs are chemotactically guided towards the cancer cells and contribute to the formation of a cancer microenvironment. The homing of BMSCs was affected by various factors. Disseminated tumour cells (DTCs) in distant organs, especially in the bone marrow, are the source of cancer metastasis and cancer relapse. DTC survival is also determined by the microenvironment. Here we aim to elucidate how cancer-educated BMSCs promote the survival of cancer cells at primary tumour sites and distant sites. We highlight the dynamic change by identifying different gene expression signatures in intratumoral BMSCs and in BMSCs that move back in the bone marrow. Intratumoral BMSCs acquire high mobility and displayed immunosuppressive effects. Intratumoral BMSCs that ultimately home to the bone marrow exhibit a strong immunosuppressive function. Cancer-educated BMSCs promote the survival of lung cancer cells via expansion of MDSCs in bone marrow, primary tumour sites and metastatic sites. These $\mathrm{Ly}_{6 \mathrm{G}} \mathrm{G}^{+} \mathrm{MDSC}$ suppress proliferation of T cells. CXCL5, nitric oxide and GM-CSF produced by cancer-educated BMSCs contribute to the formation of malignant microenvironments. Treatment with CXCL5 antibody, the iNOS inhibitor 1400w and GM-CSF antibody reduced MDSC expansion in the bone marrow, primary tumour sites and metastatic sites, and promoted the efficiency of PD-L1 antibody. Our study reveals that cancer-educated BMSCs are the component of the niche for primary lung cancer cells and DTCs, and that they can be the target for immunotherapy.
\end{abstract}

\section{Introduction}

Bone marrow mesenchymal stem cells (BMSCs) are multipotent stromal cells that can differentiate into a variety of cell types, including osteocytes, chondrocytes, adipocytes, epithelial cells and endothelial cells ${ }^{1-3}$. BMSCs have been extensively explored in functions of

\footnotetext{
Correspondence: Juanjuan Xiang (xiangjj@csu.edu.cn)

${ }^{1} \mathrm{NHC}$ Key Laboratory of Carcinogenesis and the Key Laboratory of Carcinogenesis and Cancer Invasion of the Chinese Ministry of Education, Xiangya Hospital, Central South University, Changsha, Hunan, China ${ }^{2}$ Cancer Research Institute, School of Basic Medical Science, Central South University, Changsha, Hunan, China

Full list of author information is available at the end of the article. Edited by G. Raschellà
}

immunomodulation, tissue regeneration and hematopoietic support ${ }^{4-6}$. BMSCs with robust chemotactic properties are recruited to tumours and contribute to cancer progression $^{7,8}$. Fifty-five to sixty-five per cent of injected BMSCs in mice were recovered from the bone marrow, indicating the specific bone marrow homing of systemic BMSC ${ }^{9}$. However, the homing ability of BMSCs was affected by various factors ${ }^{9,10}$. BMSCs are not only the key modulator of bone marrow environment, but also the main component of cancer microenvironment.

The behaviour of cancer cells is strongly influenced by their microenvironment ${ }^{11}$. Meanwhile, tumour cells persistently shape their microenvironment, thereby establishing an

\section{(c) The Author(s) 2019}

(c) (i) Open Access This article is licensed under a Creative Commons Attribution 4.0 International License, which permits use, sharing, adaptation, distribution and reproduction cc in any medium or format, as long as you give appropriate credit to the original author(s) and the source, provide a link to the Creative Commons license, and indicate if changes were made. The images or other third party material in this article are included in the article's Creative Commons license, unless indicated otherwise in a credit line to the material. If material is not included in the article's Creative Commons license and your intended use is not permitted by statutory regulation or exceeds the permitted use, you will need to obtain permission directly from the copyright holder. To view a copy of this license, visit http://creativecommons.org/licenses/by/4.0/. 
abnormal ecosystem. Tumour-associated fibroblasts promote tumorigenesis, whereas normal fibroblasts do not show potent tumorigenesis. These properties indicate that the tumorigenesis of inflammatory fibroblasts is due to the influence of cancer cells ${ }^{12,13}$.Tumour-stroma interactions modulate the microenvironment to be more permissive to cancer cells ${ }^{14}$. Tumour cell hierarchy, as well as multiple cellular elements in the microenvironment, co-evolve during the process of carcinogenesis ${ }^{11}$. When cancer cells leave their primary sites, enter the bloodstream and lodge into a distant organ, they display heterogeneity because of evolutionary selection ${ }^{15}$. BMSCs also evolve and differentiate in cancer microenvironments ${ }^{16,17}$. Once the BMSCs home to a cancer microenvironment in response to chemokines and cytokines secreted by cancer cells, they are 'educated' by the cancer microenvironment. BMSCs can differentiate into adipocytes, endothelial and fibroblasts in the cancer microenvironment ${ }^{1,18}$.

Increasing evidence suggests that cancer cell dissemination is an early event, sometimes taking place even before the formation of overt primary tumours ${ }^{19}$. The fate of disseminated tumour cells (DTCs) influences cancer patients outcomes ${ }^{15}$. The survival of DTCs in distant organs is determined by the formation of a pre-metastatic niche ${ }^{20}$. DTCs in distant organs have enhanced malignant features and can redisseminate ${ }^{19}$. DTCs may recirculate from the bone marrow to disseminate again, which is the source of cancer relapse. Bone marrow is a preferred site for DTCs and is considered to be a 'metastatic niche ${ }^{21}$. The bone marrow microenvironment is the niche not only for hematopoietic stem cells but also for disseminated cancer cells ${ }^{22}$.

Although the potential for undergoing differentiation of BMSCs into multiple cell type has been validated in vitro, the lack of a MSC-specific marker limits the investigation into the behaviour of BMSCs in cancer patients. In this study, we set out to establish a model that allows us to investigate the dynamic change of BMSCs and how the interaction of BMSCs and cancer cells affect cancer progression. Our study highlights the co-evolution of cancer and stromal compartments. We describe the evolution of BMSCs during the homing process and the progression of cancer by identifying two subpopulations that are distinct from the original BMSCs. We identify the subpopulations of intratumoral BMSCs (T-BMSCs), which show high motility and immunosuppressive effects, and BMSCs, which move from primary tumour sites back into the bone marrow (B-BMSCs) and are associated with an stronger immunosuppressive environment. The cancer-educated BMSCs promote the survival of cancer cells in primary and distant metastatic sites via the induction of bone marrow-derived polymorphonuclear myeloid-derived suppressor cells (PMN-MDSCs) expansion. A better understanding of the features of microenvironmental BMSCs raises the possibility that targeting specific BMSCs can help to prevent the progression of cancer in different stages.

\section{Methods \\ Cells}

Murine BMSCs from C57BL/6 mice were purchased from Cyagen Company, China. Non-small cell lung cancer (NSCLC) cell lines including H157, A549, H460 and murine Lewis lung carcinoma cells (LLCs) were cultured in RPMI-1640 medium supplemented with penicillin G $(100 \mathrm{U} / \mathrm{mL})$, streptomycin $(100 \mathrm{mg} / \mathrm{mL})$ and $10 \%$ fetal calf serum. Cells were grown at $37^{\circ} \mathrm{C}$ in a humidified atmosphere of $5 \% \mathrm{CO}_{2}$ and were routinely sub-cultured using $0.25 \%(\mathrm{w} / \mathrm{v})$ trypsin-EDTA solution. Human BMSCs were obtained from BM aspirates of non-haematological malignant tumour patients. Samples were from Xiangya Hospital, Central South University, China. The collection of the bone marrow was performed for diagnosis. The patients were informed about the sample collection and have signed informed consent forms. Collections and use of tissue samples were approved by the ethical review committees of Xiangya Hospital. Bone marrow aspirates were collected and stored in evacuated tubes containing anticoagulants. Mononuclear cells were isolated from bone marrow using Ficoll-Paque ${ }^{\mathrm{TM}}$ PLUS (Density $1.077 \pm 0.001 \mathrm{~g} / \mathrm{mL}$, GE Healthcare, 17144002). The mononuclear cells were collected and cultured in flasks. Three days after plating, the non-adherent cells were removed. When the adherent cells reached confluence, the cells were then passaged and used for the following experiments at passages 4-6. Human BMSCs and mice BMSCs are monolayer cultured in Medium For Human Mesenchymal Stem Cells (HUXMA-03011-440,cyagen). Firefly luciferase-expressing Luci-LLCs were a kind gift from Professor Wen Zhou (Cancer Research Institute, Central South University). GFP-BMSCs and RFP-LLCs were constructed by lentivirus-mediated green fluorescent protein (GFP) or red fluorescent protein (RFP) gene transduction. The lentivirus-based vector expressing GFP or RFP was purchased from Cyagen, China.

\section{Animal experiments}

Six-week-old male C57BL/6 mice were used to examine allograft tumour growth. Animal experiments were conducted following protocols approved by Central South University, China. $1 \times 10^{6}$ of murine LLC cells with or without murine BMSCs were injected subcutaneously into syngeneic C57BL/6 mice. BMSCs were injected with the same amount of LLCs. The tumours were measured daily using calipers, and their volumes were calculated using the following standard formula: length $\times$ width $^{2} \times$ 0.5 . To measure the disseminated cancer cells in the circulation and in the bone marrow, LLCs were stably transfected with red fluorescent protein RFP and BMSCs 
were stably transfected with GFP. The GFP-positive and $R F P$-positive cells were recorded after analysis on flow cytometer (BD Biosciences).

To investigate the metastatic cancer cells in vivo, the LLCs were stably transfected with the firefly luciferase gene (luci-LLCs) were subcutaneously injected with or without BMSCs into C57BL/6 mice. Non-invasive bioluminescence imaging was performed using Bruker In-Vivo Xtreme II instruments. The mice were intraperitoneally injected with $100 \mu \mathrm{l}$ of D-luciferin $(100 \mathrm{mM})$. The bioluminescence images were acquired using a charge-coupled device camera $10 \mathrm{~min}$ after injection. The primary tumours were cut to avoid signal saturation.

To investigate the chemotactic effect of CXCL5, 6-weekold male C57BL/6 mice were used. Murine RFP-positive LLC cells $\left(R F P\right.$-LLCs; $\left.1 \times 10^{6}\right)$ with murine GFP-positive BMSCs (GFP-BMSCs) were injected subcutaneously into syngeneic C57BL/6 mice. BMSCs were injected with the same amount of LLCs. Fifteen days after the inoculation of cancer cells, the tumour-bearing mice were intraperitonially injected with CXCL5 neutralizing antibody $(1 \mathrm{mg} / \mathrm{kg} /$ day, R\&D), CXCR2 antagonist $(1 \mathrm{mg} / \mathrm{kg} /$ day, selleck) or IgG control $(1 \mathrm{mg} / \mathrm{kg} /$ day, R\&D) every 3 days. The tumours were measured daily using calipers and their volumes were calculated using the following standard formula: length $\times$ width $^{2} \times 0.5$. The GFP-positive and $R F P$-positive cells were recorded after analysis on flow cytometer (BD Biosciences).

For the PD-L1 blockage efficacy experiment, 6-week-old male C57BL/6 mice were used. Murine RFP-positive LLC cells (RFP-LLCs; $1 \times 10^{6}$ ) with murine GFP-positive BMSCs (GFP-BMSCs) were injected subcutaneously into syngeneic C57BL/6 mice. BMSCs were injected with the same amount of LLCs. Fifteen days after the inoculation of cancer cells, the tumour-bearing mice were intraperitonially injected with PD-L1 antibody ( $200 \mu \mathrm{g} /$ mouse, Bioxcell), $1400 \mathrm{~W}$ $(2 \mathrm{mg} / \mathrm{kg} /$ day, Beyotime, China), CXCL5 neutralizing antibody $(1 \mathrm{mg} / \mathrm{kg} /$ day, R\&D) or granulocyte-macrophage colony-stimulating factor (GM-CSF) antibody (R\&D) every 3 days. The tumours were measured daily using calipers and their volumes were calculated using the following standard formula: length $\times$ width $^{2} \times 0.5$. The GFP-positive and RFPpositive cells were recorded after analysis on flow cytometer (BD Biosciences).

\section{Flow cytometry}

Lungs, bone marrow and cancer samples were minced, digested and homogenized. Cells were passed through a $70 \mu \mathrm{m}$ nylon filter. $\mathrm{GFP}^{+}$and $\mathrm{RFP}^{+}$cells were recorded after analysis on flow cytometer (BD Biosciences). The primary antibodies conjugated with fluorochome were used to detect, sort and purify the target antigens.

Extracellular markers included mouse Percp-cy5.5CD11b, APC-Ly6G, PE-Ly6C, APC-CD4, PE-cy7-CD8,
PE-Gr-1, Hunan PE-CXCR2 and mouse Alexa Fluor 647CXCR2 were used. All antibodies were purchased from BD Biosciences.

\section{RNA sequencing}

RNA-sequencing (RNA-seq) analysis was performed using BGISEQ-500 platform. Total RNA was isolated and cDNA library was constructed. High-quality clean reads were aligned to the human reference genome using Bowtie2. The expression levels for each of the genes were normalized as fragments per kilobase of exon model per million mapped reads (FPKM) by Expectation Maximization. The differential gene expression analysis was performed by DESeq2. Hierarchical cluster analysis based on $\log 2$ (ratios) of differentially expressed genes was performed by OmicShare. Interpreting sets of genes was based on GO term enrichment in which genes are classified depending on their functional characteristics.

\section{Cytokine array analysis}

Conditioned media was prepared as described above and applied to a RayBiotech antibody array according to the manual. After incubation with media, the containers were then placed on a shaker and washed three times with $1 \times$ Wash Buffer at room temperature. Two millilitres of diluted Streptavidin-horseradish peroxidase was then added to each membrane and incubated at room temperature with gentle shaking for $2 \mathrm{~h}$. Finally, the prepared Chemi Reagent Mix was pipetted onto each membrane. The chemiluminescent signals were captured and the intensity of the dots was quantified using a Bio-Rad ChemiDoc XRS system (Bio-Rad, CA, USA). The resulting images were analyzed using Image $J$ to measure the expression levels of the various targets. A positive control was used to normalize the results from the different membranes being compared.

\section{Cell mobility}

Cell mobility capacity was assessed using Transwell Cell Culture Inserts $(8 \mu \mathrm{m}$ pore size, BD Biosciences, New Jersey, USA) in 24-well plates. A total of $1 \times 10^{5}$ cells in $100 \mu \mathrm{l}$ of serum-free medium were added to the top chamber. The bottom well contained growth medium with $20 \%$ fetal bovine serum. Cells were incubated for $36 \mathrm{~h}$ at $37^{\circ} \mathrm{C}$ and then the cells that had invaded through the filter pores were fixed with methanol, stained with hematoxylin and observed under a microscope. The number of motive BMSCs were counted from five randomly selected $20 \times$ fields for each experiment and averaged.

\section{Real-time PCR}

Total RNA for RNA-seq experiment was used for realtime PCR to confirm the expression of genes. cDNA was synthesized from total RNA using the RevertAid First 
Strand cDNA Synthesis Kit (Thermo Scientific, Waltham, MA, USA). GAPDH was used as the endogenous control. Quantification PCR was performed according to the indications. Real-time PCR was performed using the BioRadIQ ${ }^{\mathrm{TM} 5}$ Multicolor Real-Time PCR detection System (Bio-Rad, Berkeley, CA, USA). Relative mRNA expression levels were calculated by the $2^{-\Delta \Delta \mathrm{Ct}}$ method. The primers are listed in Supplementary Table 1.

\section{Preparation of MDSCs from bone marrow and immunosuppressive assay}

The $\mathrm{Ly}_{6 \mathrm{G}}{ }^{+}$MDCSs were isolated from bone marrow. The anticoagulated bone marrow aspirate was collected from mice that received co-injection of LLC cells and BMSCs. The cell suspension was centrifuged at 12,000 r.p.m. for $5 \mathrm{~min}$. The cell pellet was resuspended in phosphate-buffered saline. The Ly6G ${ }^{+}$MDSCs were isolated by The BD FACScell sorter. The mouse spleen $\mathrm{T}$ cells were isolated with CD90.2 MicroBeads in a magnetic field (Miltenyi Biotec). The ly6G ${ }^{+}$MDSCs were co-cultured with CD90.2-positive $\mathrm{T}$ cells for 3 days. The CD4- and CD8-positive cells were measured by flow cytometry (BD Biosciences).

\section{Statistical analysis}

Data are presented as the mean \pm SD from at least three separate experiments. Statistical analyses were performed using GraphPad Prism 5 (GraphPad Software, Inc., CA, USA). Group comparisons were performed using Student's $t$-test. The survival of tumour-bearing mice was analyzed by Kaplan-Meier. A $p$-value of $<0.05$ was considered to be significant.

\section{Results}

BMSCs promote lung cancer cell growth and metastasis

To evaluate whether BMSCs promote cancer growth and facilitate the metastatic process of cancer cells, we constructed syngeneic tumour model that murine BMSCs and murine LLC cells were subcutaneously injected into C57BL/6 mice. LLCs that were stably transfected with the firefly luciferase gene (luci-LLCs) were subcutaneously injected with or without BMSCs into C57BL/6 mice. About 24 days later, LLC allograft tumours were significantly larger when the RFP-LLCs were co-transplanted with BMSCs (Fig. 1a, $p<0.05$ ). The mobility and growth of the LLCs in vivo were examined by bioluminescence imaging. To avoid the signal saturation of the primary tumour, we cut the primary tumour before the detection of in vivo tumour formation. In contrast to the mice that received injection of LLCs alone and that had little evidence of micrometastatic cells, the mice that received injection of a mixture of LLCs and BMSCs showed high luciferase activity in the lungs and bone marrow (Fig. 1b).
The number of metastatic tumour modules formed in the lungs was greater in the mixture-injection group than in the LLCs single-injection group (Fig. 1c). Hematoxylin and eosin-stained sections showed metastatic nodules in the lungs in the mixture-injection group (Supplementary Fig. 1A). Histological analysis demonstrated increases in the numbers of Tartrate-resistant acid phosphatase (TRAP)-positive cells and bone lesions (Fig. 1d). Addition of BMSCs shortened the survival of the recipient mice compared to injection of LLCs alone (Fig. 1e). These results indicate that BMSCs promote the growth and metastatic abilities of lung cancer cell.

\section{Cancer-BMSCs interaction improve capability of dissemination and homing of cancer cells and BMSCs to distant organs}

Dissemination and survival of cancer cells in the bloodstream and in distant organs are required for the formation of metastatic loci. To measure the disseminated cancer cells in the circulation and in bone marrow, RFPlabelled LLC cells (RFP-LLCs) were injected subcutaneously into C57BL/6 mice with or without GFPlabelled BMSCs (GFP-BMSCs). The flow cytometry assay revealed that circulating RFP-positive LLCs were detected in mice that received the mixture injection or LLCs injection alone, suggesting that the dissemination of cancer cells into the circulation is a frequent event. The ratio of circulating cancer cells in the mixture-injection group was greater than that in the LLC injection alone group (Fig. 2a, b). The modified ISET combined with fluorescent imaging revealed the existence of RFP-LLCs in the circulation (Supplementary Fig. 1B). The ratio of RFPpositive LLCs residing in the bone marrow and in the lungs was greater in the mixture-injection group compared with the LLC alone injection group (Fig. 2a, b). GFP-BMSCs were also detected in the circulation, in bones and in the lungs of the mice that received LLC and BMSC co-injection, indicating that intratumoral BMSCs also disseminated into the circulation (Fig. 2c, d). The number of RFP-LLCs in circulation and in bone marrow was positively correlated with the number of GFP-BMSCs (Supplementary Fig. 1C). These results indicate that cancer-educated BMSCs preferentially disseminate and home to bone marrow and lungs.

To investigate whether cancer cells affect the dissemination and homing of BMSCs, GFP-positive BMSCs were exposed to media collected from LLCs for $72 \mathrm{~h}$, followed by subcutaneous injection into mice. Flow cytometry analysis was used to track the BMSCs in circulation. We found that the BMSCs primed by LLC cells moved earlier into circulation and settled more in the lungs and bone marrow compared with non-primed BMSCs (Supplementary Fig. 1D). 


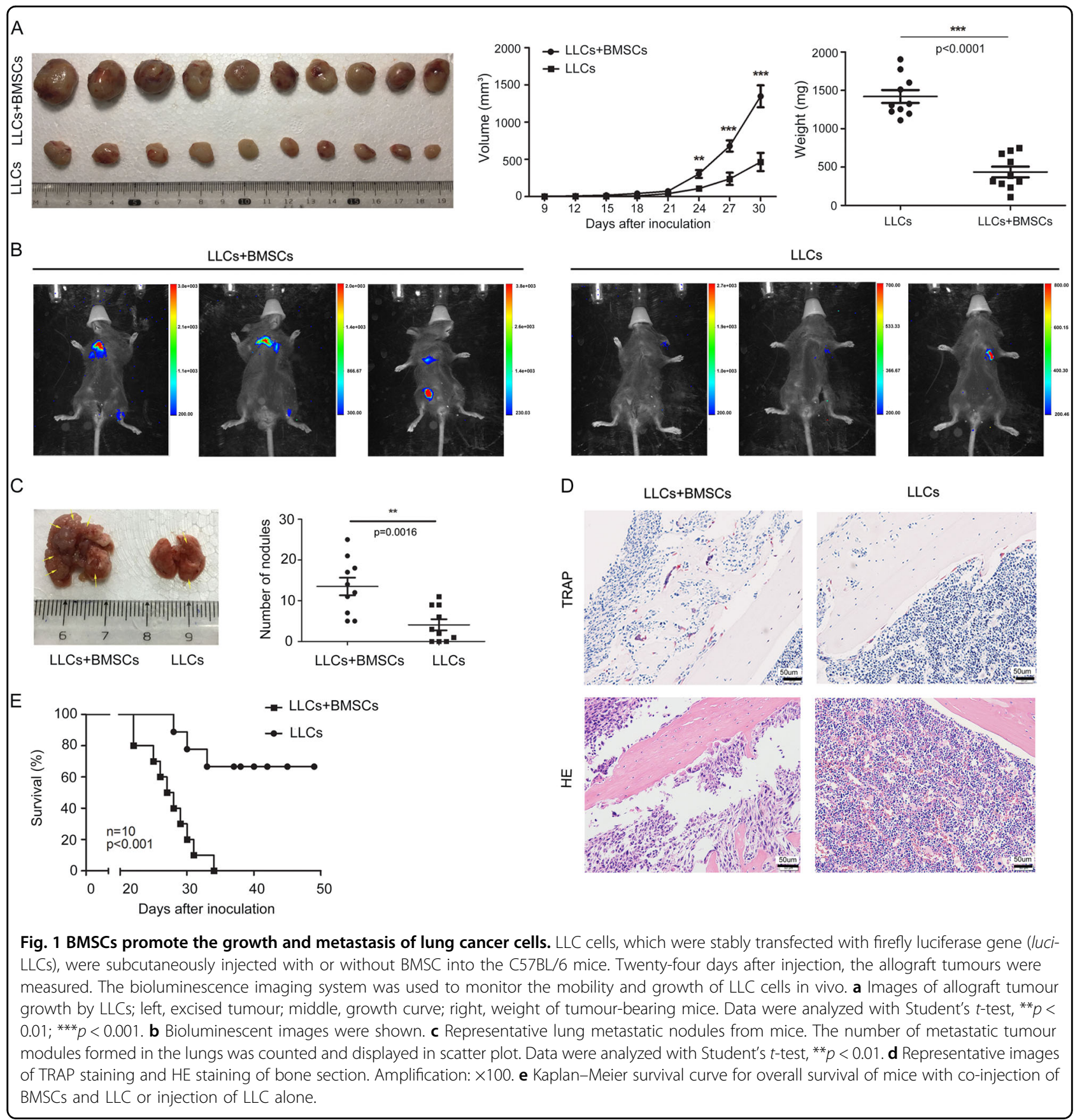

\section{Spatial evolution of BMSCs during the process of dissemination}

BMSCs are attracted to cancer sites in response to chemokines produced in the cancer microenvironment. As shown above, the intratumoral BMSCs can disseminate into the circulation and reside in bones. We then compared different transcriptomic signatures by performing RNA-seq. We subcutaneously injected the RFP-LLC cells and GFP-BMSCs into C57BL/6 mice and then collected GFP-BMSCs from the primary inoculation cancer site and from the bone marrow by flow cytometric sorting technology. The sequencing raw data are available by accession number (GSE120456). Gene expression profiling was performed on BMSCs collected from 3 samples for each group. Principal component analysis showed that the BMSCs collected from primary cancer sites (T-BMSCs) and those from the bone marrow (BBMSCs) had globally different gene expression profiles (Fig. 3a). Hierarchical clustering analysis showed discrete clustering of the BMSC subsets from cancer primary sites 


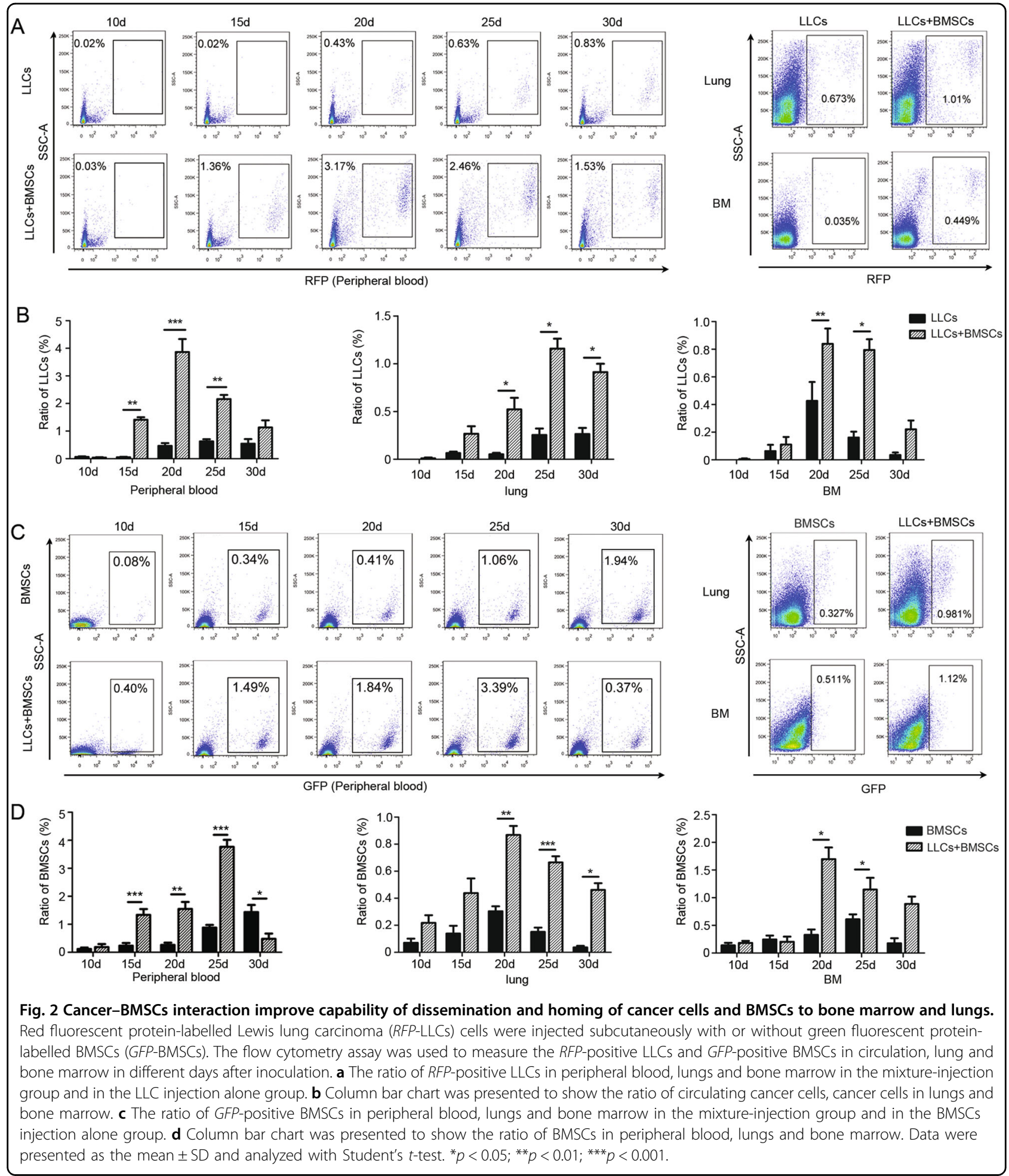

(T-BMSCs) and tumour-bearing bone marrow (BBMSCs) (Fig. 3b). Compared with intact BMSCs, intratumoral BMSCs had 1820 upregulated and 1147 downregulated genes. mRNA profiling demonstrated that the genes with higher expression in T-BMSCs were cytoskeleton-, cytokine- and immunoregulation-related genes (Fig. 3c and Supplementary Table 2). Compared with the intratumoral BMSCs, cancer-educated BMSCs moving to the bone marrow (B-BMSCs) had 1483 upregulated and 1220 downregulated genes. A Gene Ontology 

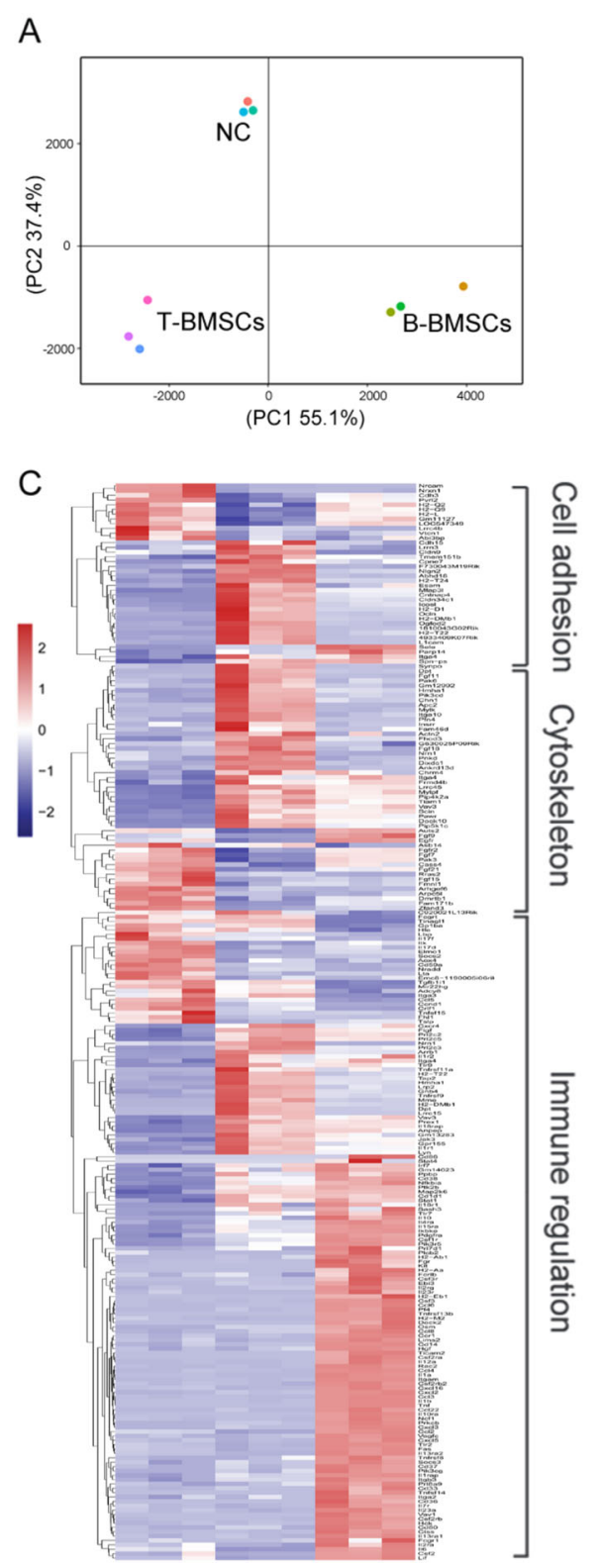

B
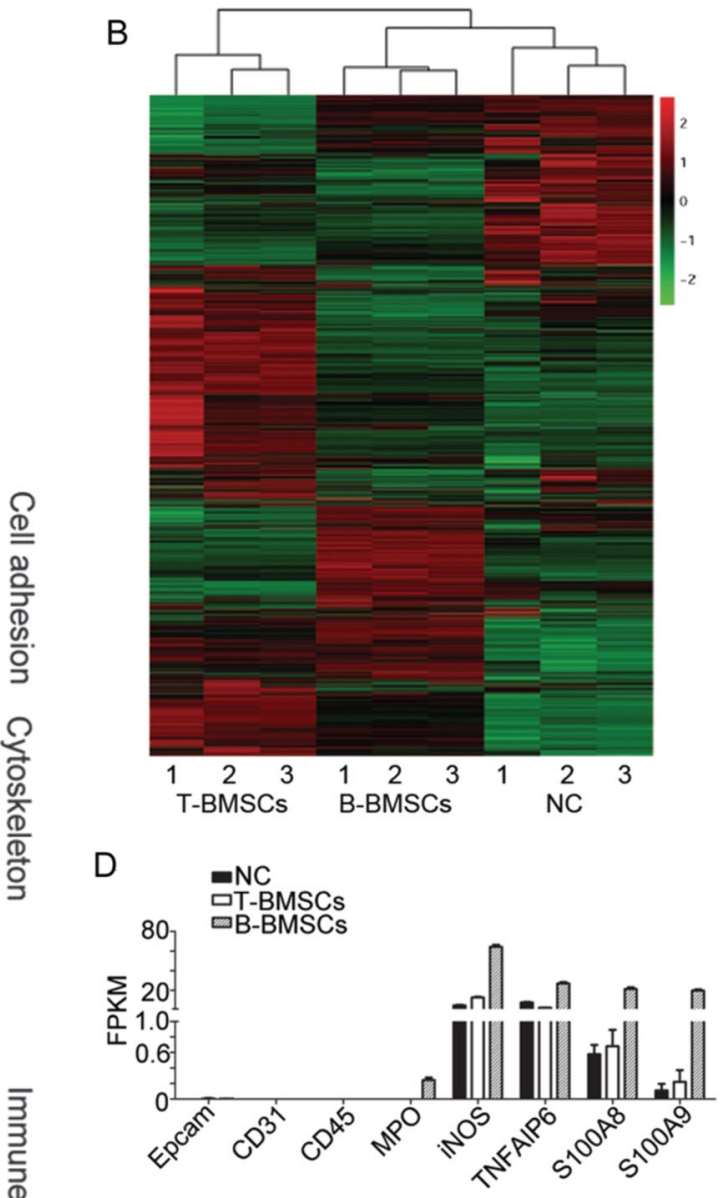

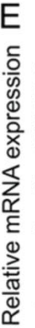

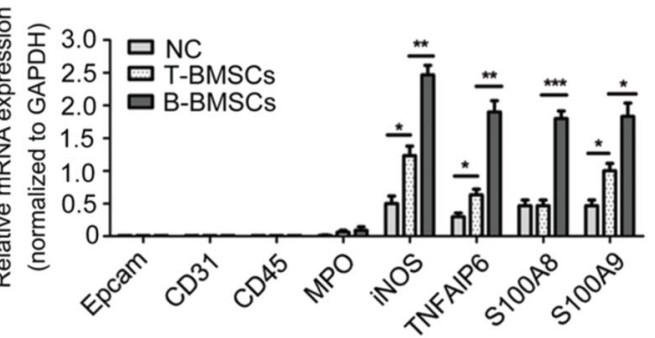

Fig. 3 Spatial evolution of BMSCs during the process of dissemination. GFP-BMSCs from the primary inoculation cancer site and bone marrow were collected by flow cytometer sorting technology. The cellular RNA from BMSCs was subjected to RNA-sequencing. a Principal component analysis plot. $\mathbf{b}$ Hierarchical clustering analysis of gene expression. Clustering was performed on differentially expressed genes at FDR $<0.05$ from nine samples of BMSCs. Columns represent individual samples and rows represent each gene. Each cell in the matrix represents the expression level of a gene feature in an individual sample. Red and green in cells reflect high and low expression levels, respectively, as indicated in the scale bar (log2-transformed scale). c Hierarchical clustering analysis on differentially expressed genes related to selected functions. Columns represent individual samples and rows represent each gene. $\mathbf{d}$ Expression of genes enriched in the osteoclast differentiation and immunosuppressive pathway quantified by RNA-Seq. FPKM for selected gene transcripts obtained by RNA-Seq. e The expression of genes was validated by real-time PCR. Data were analyzed with Student's $t$-test, ${ }^{*} p<0.05 ;{ }^{* *} p<0.01 ;{ }^{* * *} p<0.001$.

(GO) enrichment analysis demonstrated that the genes with higher expression in B-BMSCs were enriched in immune system process-related genes (Fig. 3c). BMSCs collected from cancer primary sites or from bone marrow were epithelial marker (EPCAM), endothelial marker
(CD31), hematopoietic marker (CD45 and CD34) and neutrophil marker $(M P O)$ negative. The upregulated genes were enriched in the osteoclast differentiation and immunosuppressive pathway in B-BMSCs. The T-BMSCs and B-BMSCs expressed many immunologic suppression 
molecules including ${ }^{2 N O S^{23}}{ }^{2}$ TNFAIP $6^{24}$ and S100A8/ $S 100 A 9^{25}$ (Figs. 3d). The expressions were validated by real-time PCR (Fig. 3e).

\section{Cancer-educated BMSCs attract cancer cells into circulation through CXCL5/CXCR2}

We next determined whether cancer-educated BMSCs may attract cancer cells into the circulation. We cocultured BMSCs with LLC cells in vitro and intravenously injected the cancer-educated GFP-BMSCs into C57BL/6 mice, followed by intravenous injection of RFP- LLCs 3 days later. Compared with the control BMSCs, intravenously injected cancer-educated BMSCs resided more in lungs and bone marrow (Fig. 4a and Supplementary Fig. 2A). Intravenous injection of cancer cell-primed BMSCs attracted more LLCs to the lungs and bone (Fig. 4b and Supplementary Fig. 2B). We then measured differential chemokine secretion, which might account for the differences in the numbers of cancer cells mobilized into the circulation by cancer cell-educated BMSCs vs. non-educated BMSCs. We collected conditional media from human BMSCs alone or BMSCs co-cultured with A549 lung cancer cells. A number of chemokines were significantly upregulated in cancer cell-educated BMSCs compared with non-educated BMSCs, including CXCL5 and CCL5 (Fig. 4c). Having analyzed the gene expression patterns determined by RNA-seq in T-BMSCs and BBMSCs, we found that T-BMSCs and B-BMSCs exhibited high expression of the genes coding for the chemokines CXCL5, but not CCL5 (Fig. 4d). The expressions of CXCL5 and CCL5 were validated by real-time PCR (Fig. 6a). The lung cancer A549 cells, H157 cells, H460 cells and LLCs were shown to be CXCL5 receptor CXCR2 positive (Supplementary Fig. 2C). Recombinant CXCL5 showed a strong chemotactic effect on A549 cells, H157 cells, H460 cells and LLCs (Fig. 4e and Supplementary Fig. 2D, E, F). The chemotactic effects were reversed by anti-CXCL5 neutralizing antibody or CXCR2 antagonist (Fig. 4e and Supplementary Fig. 2D, E, F). The chemotactic role of CXCL5 derived from cancer-educated BMSCs on LLCs was investigated in C57BL/6 mice. C57BL/6 mice were subcutaneously injected with RFPLLCs and BMSCs. Fifteen days later, intraperitoneal injection of anti-CXCL5 neutralizing antibody or CXCR2 antagonist decreased the recruitment of subcutaneously injected RFP-LLC cells into the circulation and significantly prolonged the survival of tumour-bearing mice (Fig. 4f, g and Supplementary Fig. 3A).Treatment with anti-CXCL5 neutrlizing antibody and CXCR2 blockage also reduced the growth of primary tumours, indicating that CXCL5/CXCR2 could have effect on tumour growth other than chemotactic effects (Supplementary Fig. 3B, C, D).

\section{Cancer-educated BMSCs induce the expansion of PMN- MDSCs in bone marrow}

To explore the mechanism of how cancer-educated BMSCs promote the survival of lung cancer cells in the primary tumour sites and in bone marrow, we studied immunosuppressive cells in bone marrow in LLC tumourbearing mice with or without BMSCs. A prominent expansion of $\mathrm{Gr}-1^{+} / \mathrm{CD} 11 \mathrm{~b}^{+}$cells in the bone marrow was found in both groups of tumour-bearing mice, especially in mice with co-injection of LLC cells and BMSCs (Fig. 5a). We further found that among the $\mathrm{Gr}-1^{+} / \mathrm{CD} 11 \mathrm{~b}^{+}$cells, the

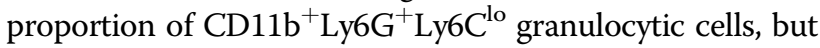
not $\mathrm{CD} 11 \mathrm{~b}^{+} \mathrm{Ly} 6 \mathrm{C}^{+}$monocytic cells, was significantly increased (Fig. 5b and Supplementary Fig. 4A). The pro-

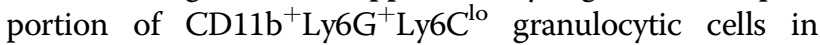
spleen was also significantly increased (Supplementary Fig. 4B). Intracavitary injection of cancer-educated BMSCs into the bone marrow resulted in the expansion of CD11b ${ }^{+}$Ly6G $^{+}$Ly6C $^{\text {lo }}$ cells (Fig. 5c). Ly6G ${ }^{+}$MDSC subpopulations were isolated from bone marrow by magnetic activated cell sorting. These $\mathrm{CD}_{11} \mathrm{~b}^{+} \mathrm{Ly}_{6 \mathrm{G}}{ }^{+} \mathrm{Ly} 6 \mathrm{C}^{\mathrm{lo}}$ granulocytic cells potently inhibited $\mathrm{CD}^{+}$and $\mathrm{CD} 8^{+}$T-cell proliferation in vitro (Fig. $5 \mathrm{~d}$ ). $\mathrm{CD}^{+}$and $\mathrm{CD}^{+}{ }^{+} \mathrm{T}$ cells in the bone marrow and spleen were obviously reduced in mice with co-injection of LLC cells and BMSCs in contrast to the mice with LLC injection alone (Fig. 5e and Supplementary Fig. 4C). These populations of $\mathrm{CD}_{11 \mathrm{~b}^{+} \mathrm{Ly} 6-}$ $\mathrm{G}^{+} \mathrm{Ly} 6 \mathrm{C}^{\mathrm{lo}}$ cells fit the criteria of PMN-MDSC.

\section{PMN-MDSC depletion enhances efficacy of anti-PD-L1 treatment}

We then performed real-time PCR to analyse the expressions of genes that have been implicated in MDSC expansion, including CXCL5, G-CSF, IL-6, IL-1, GM-CSF, and $i N O S$ in T-BMSCs and B-BMSCs (Fig. 6a). We found that iNOS, GM-CSF and chemokine CXCL5 were upregulated in T-BMSCs and B-BMSCs (Figs. 6a and 3e). We speculate that cancer-educated BMSCs remodelled the cancer microenvironment through these MDSC-related molecules. C57BL/6 mice were subcutaneously injected with RFP-LLCs and BMSCs. Fifteen days after inoculation, intraperitoneal injection of CXCL5 antibody, GMCSF antibody or iNOS antagonist $1400 \mathrm{~W}$ dramatically reduced the accumulation of PMN-MDSCs in the bone marrow, lungs and primary tumour sites compared with IgG-negative control (Fig. 6b). It demonstrated that cancer-educated BMSCs remodel the microenvironment in bone marrow, primary tumour sites and lungs through MDSC-related molecules.

Although a lot of evidences that PD-1/PD-L1 blockage has been shown to be helpful in treatment of advanced lung cancer patients, immunosuppression and immune evasion decreased its clinical efficacy ${ }^{26-28}$. We then 


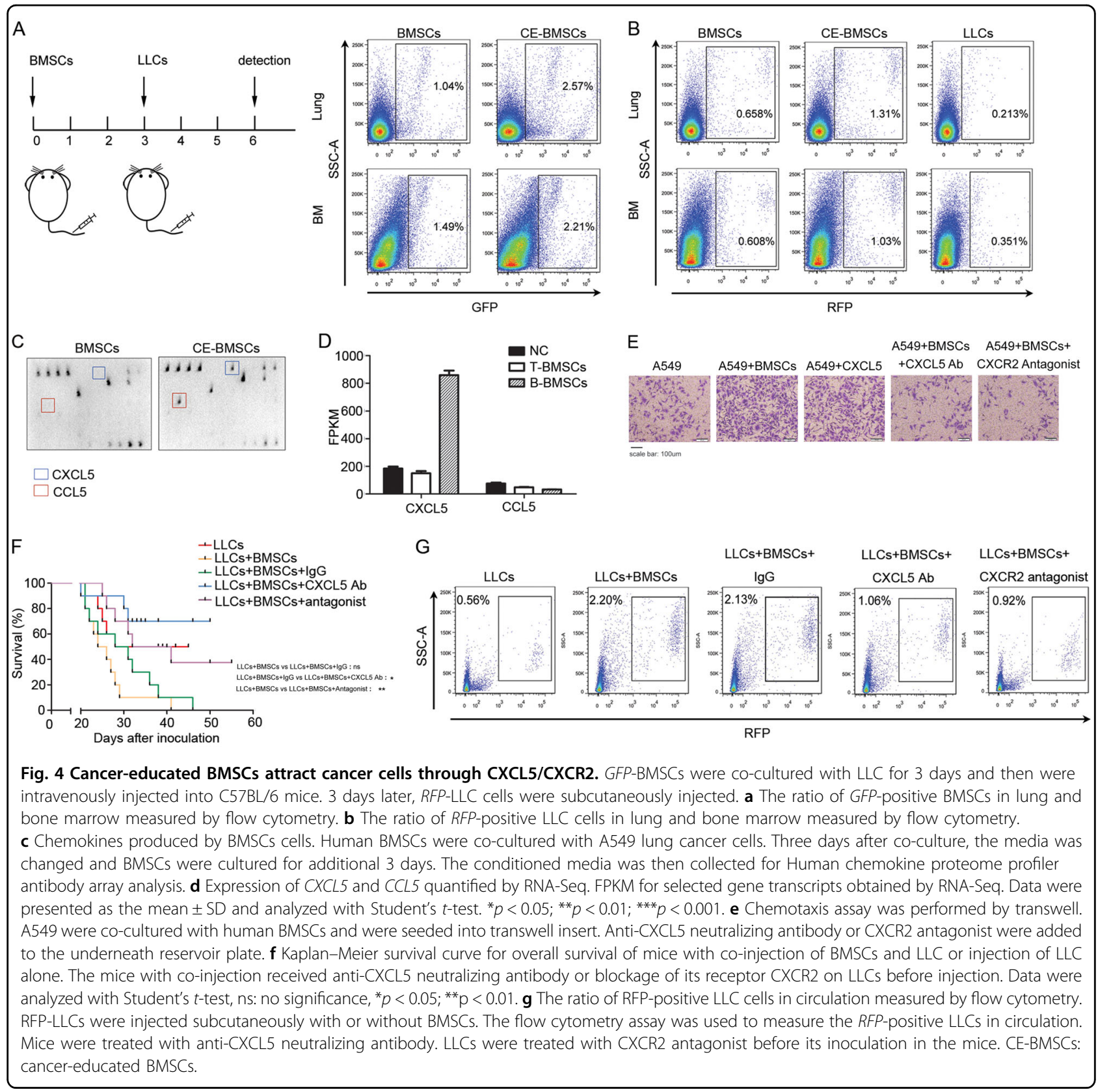

sought to investigate if PMN-MDSC depletion enhances efficacy of PD-L1 blockage. C57BL/6 mice were subcutaneously injected with RFP-LLCs and BMSCs. Fifteen days after inoculation, the tumour-bearing mice were intraperitonoally injected with anti-PD-L1 mAb. AntiPD-L1 mAb reduced the primary tumour growth and PMN-MDSCs in primary tumour sites (Fig. 6b, c and Supplementary Fig. 5A-C). In combination with the antiCXCL5 mAb, $1400 \mathrm{~W}$ or anti-GM-CSF mAb, anti-PD$\mathrm{L} 1 \mathrm{mAb}$ reduced PMN-MDSC accumulation in the primary tumours, bone marrow and the lungs more significantly than anti-PD-L1 mAb treatment alone or anti-CXCL5
$\mathrm{mAb}, 1400 \mathrm{~W}$ or anti-GM-CSF mAb treatment alone (Fig. 6b, c). The combination of CXCL5 antibody, $1400 \mathrm{~W}$ or GM-CSF antibody with anti-PD-L1mAb resulted in increased number of $\mathrm{T}$ cells in primary tumour sites (Supplementary Fig. 5D, F). The combination of CXCL5 antibody, $1400 \mathrm{~W}$ or GM-CSF antibody with anti-PD-L1 mAb reduced primary tumour growth and RFP-positive LLCs in lungs and prolonged the survival of cancer bearing mice compared with PD-L1 antibody alone, indicating that MDSC depletion can enhance the efficacy of immunotherapy (Fig. 6d and Supplementary Fig. 5A, B, E, F). 


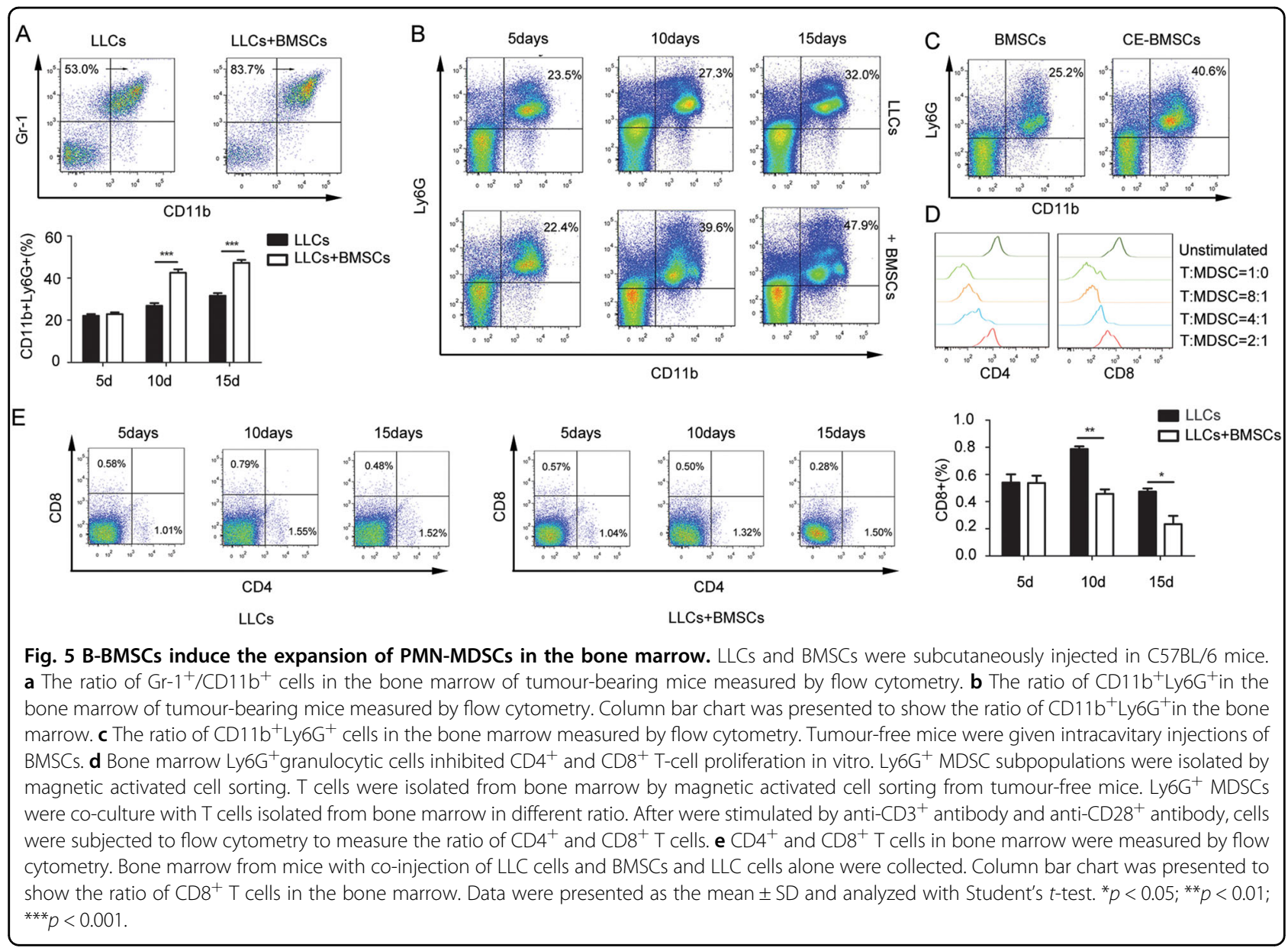

\section{Discussion}

The present work aimed at providing a better understanding of the roles of stromal cells in cancer cell growth and metastasis. We found a spatial evolution of BMSCs during the process of dissemination. We identified two types of BMSCs, each exhibiting different characteristics in mobility and immunologic regulation. T-BMSCs, which reside in the primary cancer, are highly mobile and immunosuppressive. B-BMSCs, which move from the primary cancer to the bone marrow, acquire the adverse characteristic of immunologic inhibition. The immunosuppressive molecules produced by cancer-educated BMSCs induce expansion of PMN-MDSCs and affect the efficacy of PD-L1inhibitory therapy (Fig. 6e).

During cancer progression, novel genotypic and phenotypic variants emerge via gene mutation or changes in gene expression ${ }^{29}$. Tumour cells and their stroma coevolve $^{30}$. The stroma evolves as a direct response to stress. In this study, we clarified the spatial evolution of BMSCs during cancer progression. The classification of cancereducated BMSCs is based on gene expression patterns. These BMSC variants facilitate the adaptive evolution of cancer cells. The stroma cells affect not only the primary cancer cells but also metastatic cancer cells by remodelling the distant organs.

In the bone marrow niche, hematopoietic stem cells and DTCs usually remain in the quiescent G0 state ${ }^{31}$. Although the dissemination of cancer cells occurred much earlier than we expected, DTCs in the bone marrow are not immediately competent to initiate growth ${ }^{32}$. Circulating Tumor Cells (CTCs) in the circulation have a short half-life, persisting for only $1-2 \mathrm{~h}$. Most CTCs die in the circulation as a result of shear stress and/or anoikis ${ }^{33,34}$.

Growth factors and neovasculature in the bone marrow affect the reactivation of dormant cancer cells. The dormant DTCs may be reactivated by the osteoclast-mediated release of bone-derived growth factors ${ }^{35}$. When blood vessels begin to sprout, the new tips produce molecules that transform dormant cancer cells into metastatic tumours ${ }^{36}$. This process transforms a dormant niche into a metastatic niche. A subpopulation of BMSCs with both endothelial and pericytic cell surface markers suppresses the homing of cancer cells to the bone marrow ${ }^{31}$. Although it has been long known that BMSCs are an important component of the hematopoietic stem cell niche, no specific markers have been identified. BMSCs are heterogeneous and their surface 


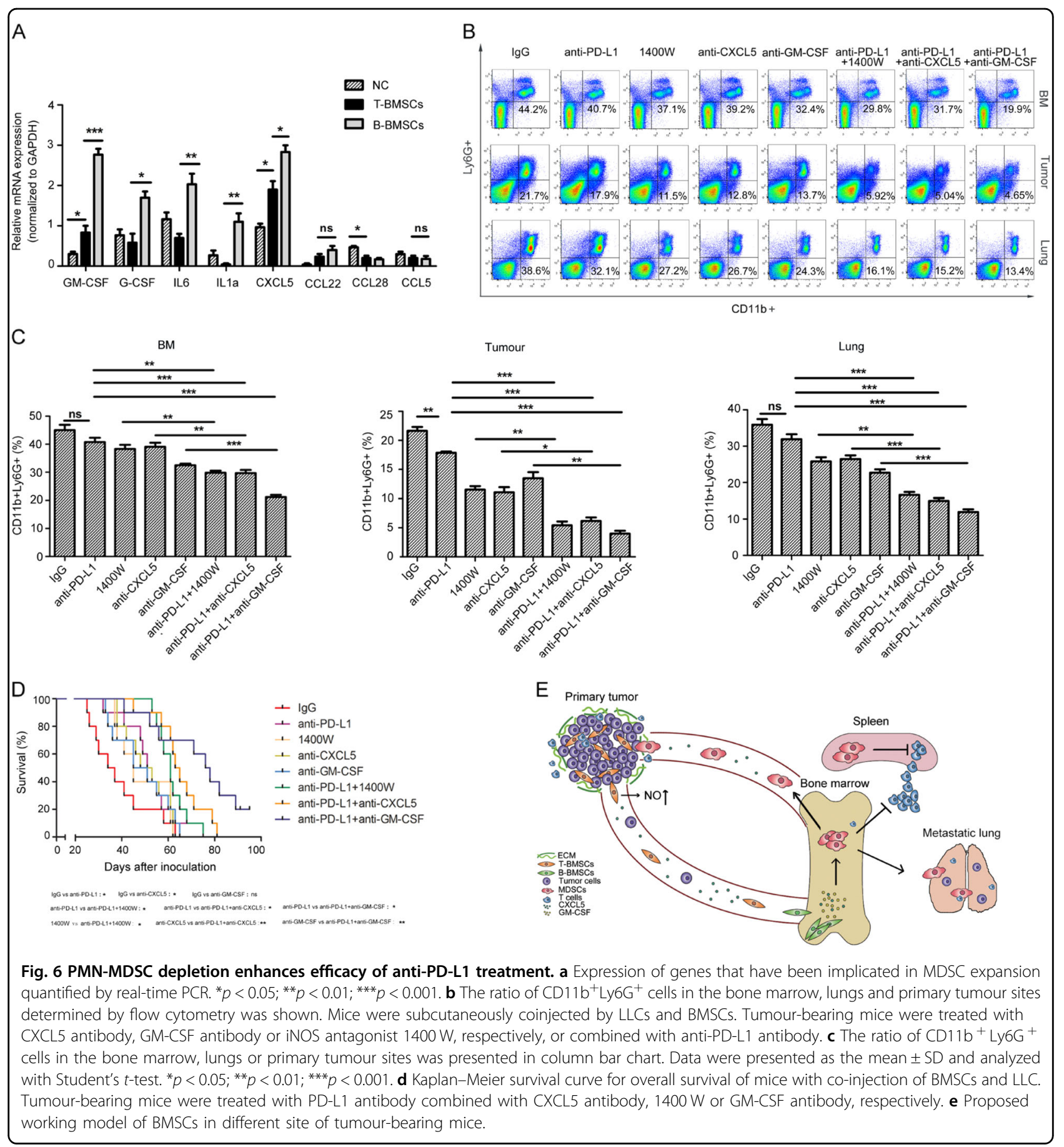

markers and behaviour are constantly changing. In contrast to the BMSCs that reside in the bone marrow, the BMSCs that reside in the primary cancer sites recruited from the bone marrow showed marked and distinct changes in their gene profiles and other features. In contrast to the original BMSCs, cancer-educated BMSCs that move back into the bone marrow showed characteristics of osteoclasts and immune suppressive cells. These subtypes of BMSCs secreted specific cytokines and chemokines, increasing the recruitment of cancer cells into circulation and bone marrow and increasing their half-life.

MDSCs are generated in the bone marrow from common myeloid progenitor cells. The accumulation and expansion of MDSCs in the bone marrow of tumourbearing hosts was reported in many studies ${ }^{37-39}$. There are two major populations of MDSCs: PMN-MDSCs and M-MDSCs. In most types of cancer, PMN-MDSCs represent $70-80 \%$ of the total MDSC population ${ }^{40}$. 
Expansion of PMN-MDSCs in the bone marrow, primary tumour sites and metastatic sites mediated by cancereducated MSCs was observed in our study. GM-CSF ${ }^{41}$, granulocyte-CSF ${ }^{42}$, and macrophage- $\mathrm{CSF}^{43}$ are responsible for the development of MDSCs. The pathologic activation of MDSCs in this study was induced by persistent stimulation coming from cancer-educated MSCs. Myeloid cells generated under these conditions are unable to differentiate effectively into mature myeloid cells ${ }^{40}$. PMN-MDSCs and neutrophils share a similar phenotype and morphology ${ }^{40}$. PMN-MDSCs induced by cancereducated MSCs suppress T-cell function, a mechanism by which cancer cells protect themselves from elimination by the immune system. MDSCs also play important roles in limiting the effect of cancer immunotherapeutics ${ }^{40}$. The roles of MDSCs in limiting the anti-tumour immune response and the effectiveness of immune checkpoint inhibitors are increasingly apparent. Suppression of MDSCs by GM-CSF antibodies or $1400 \mathrm{~W}$ enhanced the anti-cancerous effect of PD-L1 antibodies.

\section{Acknowledgements}

This work was supported by National Natural Science Foundation, China (grant numbers 81472695 and 81773147), Strategic Priority Research Program of Central South University (ZLXD2017004), Key Research and Development Program of Hunan (2019SK2253); and National Training and Research Base for Talents of principles of carcinogenesis foundation (111 project: 111-2-12).

\section{Author details \\ ${ }^{1} \mathrm{NHC}$ Key Laboratory of Carcinogenesis and the Key Laboratory of Carcinogenesis and Cancer Invasion of the Chinese Ministry of Education Xiangya Hospital, Central South University, Changsha, Hunan, China. ${ }^{2}$ Cancer Research Institute, School of Basic Medical Science, Central South University, Changsha, Hunan, China. ${ }^{3}$ Hunan Cancer Hospital, The Affiliated Cancer Hospital of Xiangya School of Medicine, Central South University, Changsha, Hunan, China. ${ }^{4}$ Hunan Key Laboratory of Nonresolving Inflammation and Cancer, Changsha, Hunan 410013, China. ${ }^{5}$ Department of Pathology, The Second Xiangya Hospital, Central South University, Changsha, Hunan 410013, China. ${ }^{6}$ Department of Thoracic Surgery, The Second Xiangya Hospital, Central South University, Changsha, Hunan 410013, China. ${ }^{7}$ Department of Spinal Surgery, The Second Xiangya Hospital, Central South University, Changsha, Hunan 410013, China}

\section{Conflict of interest}

The authors declare that they have no conflict of interest.

\section{Publisher's note}

Springer Nature remains neutral with regard to jurisdictional claims in published maps and institutional affiliations.

Supplementary Information accompanies this paper at (https://doi.org/ 10.1038/s41419-019-2149-1).

Received: 22 October 2019 Accepted: 12 November 2019 Published online: 09 December 2019

\section{References}

1. Jiang, C. et al. HIF-1A and C/EBPs transcriptionally regulate adipogenic differentiation of bone marrow-derived MSCs in hypoxia. Stem Cell Res. Ther. $\mathbf{6}$ 21 (2015).
2. Zhang, Y. et al. Mesenchymal stem cells alleviate bacteria-induced liver injury in mice by inducing regulatory dendritic cells. Hepatology 59, 671-682 (2014).

3. Shi, Y. et al. Mesenchymal stem cells: a new strategy for immunosuppression and tissue repair. Cell Res. 20, 510-518 (2010).

4. Sugino, N., Ichinohe, T., Takaori-Kondo, A., Maekawa, T. \& Miura, Y. Pharmacological targeting of bone marrow mesenchymal stromal/stem cells for the treatment of hematological disorders. Inflamm. Regen. 37, 7 (2017).

5. Wang, L. et al. Osteoblast-induced osteoclast apoptosis by fas ligand/FAS pathway is required for maintenance of bone mass. Cell Death Differ. 22 1654-1664 (2015).

6. Wang, G. et al. Kynurenic acid, an IDO metabolite, controls TSG-6-mediated immunosuppression of human mesenchymal stem cells. Cell Death Differ. $\mathbf{2 5}$ 1209-1223 (2018)

7. Krueger, T. E., Thorek, D. L. J., Meeker, A. K., Isaacs, J. T. \& Brennen, W. N. Tumorinfiltrating mesenchymal stem cells: Drivers of the immunosuppressive tumor microenvironment in prostate cancer? The Prostate 79, 320-330 (2018).

8. Karnoub, A. E. et al. Mesenchymal stem cells within tumour stroma promote breast cancer metastasis. Nature 449, 557-563 (2007).

9. Rombouts, W. J. \& Ploemacher, R. E. Primary murine MSC show highly efficient homing to the bone marrow but lose homing ability following culture. Leukemia 17, 160-170 (2003).

10. Sohni, A. \& Verfaillie, C. M. Mesenchymal stem cells migration homing and tracking. Stem Cells Int. 2013, 130763 (2013).

11. Korkaya, H., Liu, S. \& Wicha, M. S. Breast cancer stem cells, cytokine networks, and the tumor microenvironment. J. Clin. Invest. 121, 3804-3809 (2011).

12. Polyak, K, Haviv, I. \& Campbell, I. G. Co-evolution of tumor cells and their microenvironment. Trends Genet. 25, 30-38 (2009).

13. $\mathrm{Hu}, \mathrm{M}$. et al. Regulation of in situ to invasive breast carcinoma transition. Cancer Cell 13, 394-406 (2008)

14. Finger, E. C. \& Giaccia, A. J. Hypoxia, inflammation, and the tumor microenvironment in metastatic disease. Cancer Metastasis Rev. 29, 285-293 (2010).

15. Fluegen, G. et al. Phenotypic heterogeneity of disseminated tumour cells is preset by primary tumour hypoxic microenvironments. Nat. Cell Biol. 19, 120-132 (2017)

16. Mishra, P. J. et al. Carcinoma-associated fibroblast-like differentiation of human mesenchymal stem cells. Cancer Res. 68, 4331-4339 (2008).

17. Quante, M. et al. Bone marrow-derived myofibroblasts contribute to the mesenchymal stem cell niche and promote tumor growth. Cancer Cell 19, 257-272 (2011).

18. Zhang, L. et al. Tumor-conditioned mesenchymal stem cells display hematopoietic differentiation and diminished influx of Ca2+. Stem Cells Dev. 21, 1418-1428 (2012).

19. Kang, Y. \& Pantel, K. Tumor cell dissemination: emerging biological insights from animal models and cancer patients. Cancer Cell 23, 573-581 (2013).

20. Murgai, M. et al. KLF4-dependent perivascular cell plasticity mediates premetastatic niche formation and metastasis. Nat. Med. 23, 1176-1190 (2017).

21. Shiozawa, Y., Eber, M. R., Berry, J. E. \& Taichman, R. S. Bone marrow as a metastatic niche for disseminated tumor cells from solid tumors. Bonekey Rep. 4, 689 (2015).

22. Kumar, R. Godavarthy, P. S. \& Krause, D. S. The bone marrow microenvironment in health and disease at a glance. J. Cell Sci. 131, pii: jcs201707 (2018).

23. Ekmekcioglu, S., Grimm, E. A. \& Roszik, J. Targeting iNOS to increase efficacy of immunotherapies. Hum. Vaccine Immunother. 13, 1105-1108 (2017).

24. Mittal, M. et al. TNFalpha-stimulated gene-6 (TSG6) activates macrophage phenotype transition to prevent inflammatory lung injury. Proc. Natl Acad. Sci. USA 113, E8151-E8158 (2016).

25. Arora, A. et al. Serum biomarkers identification by ITRAQ and verification by MRM: S100A8/S100A9 levels predict tumor-stroma involvement and prognosis in Glioblastoma. Sci. Rep. 9, 2749 (2019)

26. Wikie, K. P. \& Hahnfeldt, P. Tumor-immune dynamics regulated in the microenvironment inform the transient nature of immune-induced tumor dormancy. Cancer Res. 73, 3534-3544 (2013).

27. Wang, Y., Zhang, X., Yang, L., Xue, J. \& Hu, G. Blockade of CCL2 enhances immunotherapeutic effect of anti-PD1 in lung cancer. J. Bone Oncol. 11, 27-32 (2018).

28. Weber, R. et al. Myeloid-derived suppressor cells hinder the anti-cancer activity of immune checkpoint inhibitors. Front. Immunol. 9, 1310 (2018).

29. Brock, A., Chang, H. \& Huang, S. Non-genetic heterogeneity-a mutationindependent driving force for the somatic evolution of tumours. Nat. Rev. Genet. 10, 336-342 (2009). 
30. Li, H., Fan, X. \& Houghton, J. Tumor microenvironment: the role of the tumor stroma in cancer. J. Cell Biochem. 101, 805-815 (2007).

31. Rossnagl, S. et al. A Subpopulation of stromal cells controls cancer cell homing to the bone marrow. Cancer Res. 78, 129-142 (2018).

32. Aguirre-Ghiso, J. A., Bragado, P. \& Sosa, M. S. Metastasis awakening: targeting dormant cancer. Nat. Med. 19, 276-277 (2013).

33. Vanharanta, S. \& Massague, J. Origins of metastatic traits. Cancer Cell 24, 410-421 (2013).

34. Meng, S. et al. Circulating tumor cells in patients with breast cancer dormancy. Clin. Cancer Res. 10, 8152-8162 (2004).

35. Gnant, M. \& Hadji, P. Prevention of bone metastases and management of bone health in early breast cancer. Breast Cancer Res. 12, 216 (2010).

36. Ghajar, C. M. et al. The perivascular niche regulates breast tumour dormancy. Nat. Cell Biol. 15, 807-817 (2013).

37. Porembka, M. R. et al. Pancreatic adenocarcinoma induces bone marrow mobilization of myeloid-derived suppressor cells which promote primary tumor growth. Cancer Immunol. Immunother. 61, 1373-1385 (2012).
38. Capietto, A. H. et al. Down-regulation of PLCgamma2-beta-catenin pathway promotes activation and expansion of myeloid-derived suppressor cells in cancer. J. Exp. Med. 210, 2257-2271 (2013).

39. Kumar, V., Patel, S., Tcyganov, E. \& Gabrilovich, D. I. The nature of myeloidderived suppressor cells in the tumor microenvironment. Trends Immunol. 37, 208-220 (2016).

40. Condamine, T., Mastio, J. \& Gabrilovich, D. I. Transcriptional regulation of myeloid-derived suppressor cells. J. Leukoc. Biol. 98, 913-922 (2015).

41. Bayne, L. J. et al. Tumor-derived granulocyte-macrophage colony-stimulating factor regulates myeloid inflammation and $\mathrm{T}$ cell immunity in pancreatic cancer. Cancer Cell 21, 822-835 (2012).

42. Okazaki, T. et al. Granulocyte colony-stimulating factor promotes tumor angiogenesis via increasing circulating endothelial progenitor cells and Gr1 +CD11b+ cells in cancer animal models. Int. Immunol. 18, 1-9 (2006).

43. Menetrier-Caux, C. et al. Inhibition of the differentiation of dendritic cells from CD34(+) progenitors by tumor cells: role of interleukin-6 and macrophage colony-stimulating factor. Blood 92, 4778-4791 (1998). 